\title{
La paz como ideal político
}

MARÍA MÓNICA PARRA*

CARLOS MAURICIO GUERRERO**

Toda política debe doblar su rodilla ante el derecho, si bien puede esperar a que le sea dado llegar, aunque lentamente, a un nivel en el que pueda brillar con firmeza.

(Kant, 1795)

\section{INTRODUCCIÓN}

En este diálogo político sobre la paz, desde una perspectiva Kantiana, académicos colombianos destacados en este campo han discutido acerca de la pertinencia, o impertinencia, de la filosofia del pensador alemán respecto de la realidad del conflicto y la paz en nuestro país. En este artículo, el semillero de investigación realiza un acercamiento al pensamiento de los profesores Rubén Jaramillo Vélez, Manuel Guillermo Rodríguez, Andrés Saldarriaga y Carlos Patarroyo.

Por un lado, Rubén Jaramillo Vélez, profesor de la Universidad Nacional, realiza un seguimiento de los antecedentes de la "paz perpetua" y resalta la importancia de tener una "política realista" como salida al proyecto inacabado de la paz que establecía Kant. Por el otro, Manuel Guillermo Rodríguez, destacado académico en el ámbito de la filosofía

* Estudiante de la Facultad de Filosofía y Letras de la Universidad Santo Tomás -Sede Bogotá-. Correo electrónico: mariaparrat@usantotomas.edu.co.

** Estudiante de la Facultad de Filosofía y Letras de la Universidad Santo Tomás -Sede Bogotá-. Correo electrónico: carlosguerreroo@usantotomas.edu.co. 
política kantiana, subraya la incidencia que tienen las revoluciones en las acciones políticas de quienes gobiernan, en la constitución que los rige y en los proyectos que están llevando a cabo. A su vez, Andrés Saldarriaga, profesor de la Universidad de Antioquia, relaciona los aportes de Kant al concepto de la paz con la realización de esta a nivel mundial, teniendo en cuenta las condiciones internas que debe cumplir un Estado para hacer parte de tal proyecto.Y finalmente, Carlos Patarroyo, profesor de la Universidad del Rosario, habilita las reflexiones de Kant en el contexto colombiano actual, pues cree firmemente en el progreso de la humanidad que guía sus acciones bajo ideas regulativas como la paz, aunque le preocupa la presión externa que se ejerce sobre los diálogos en la Habana bajo fuerzas opuestas que pueden comprometer su desarrollo.

Tal como se ve en esta pequeña introducción, en la posición de los cuatro profesores colombianos, cada uno trata de abordar el tema de la paz a partir de diferentes perspectivas, direccionadas hacia un mismo punto: la posibilidad de estudiar la problemática interna desde los postulados de Kant acerca de la paz. Se abordará a continuación un marco general de algunos conceptos kantianos en torno al proyecto de la paz, los cuales permitirán referenciar conceptualmente los enfoques de los cuatro filósofos colombianos que irán apareciendo según como los anunciamos en esta sección; en la segunda parte de este artículo daremos cuenta del pensamiento de nuestros cuatro filósofos y, finalmente, el semillero realizará un análisis aplicativo de estas reflexiones en el contexto de los diálogos de la Habana.

Pero antes de adelantar dicho plan de trabajo, debe señalarse que este artículo hace parte de los resultados de la investigación titulada "Conflicto y paz en Colombia: lecturas kantianas", que entre febrero de 2014 y marzo de 2015 realizaron los integrantes del semillero "EnKantados: Kant y nosotros", adscrito al grupo de investigación "Estudios en pensamiento filosófico en Colombia y América Latina- Bartolomé de Las Casas" de la Facultad de Filosofia y Letras de la Universidad Santo Tomás. En el marco de este proyecto, elaborado con el apoyo financiero del Fondo General de Investigación (FODEIN) de la Unidad de Investigación en la convocatoria interna 2014 - 2015, los avances del texto fueron expuestos en el "XIV Coloquio de Estudiantes de Filosofia" de la Universidad de San Buenaventura (8 y 9 de mayo de 2014), en el "VII Encuentro Tomasino de 
Investigadores en Filosofia y Cultura" de la Facultad de Filosofia y Letras de la USTA (13 y 14 de mayo de 2014) y en el "II Encuentro Nacional de Grupos y Semilleros:Visibilización de la Investigación usta Colombia" (27 de octubre de 2014). Asimismo, en el desarrollo del proyecto se realizó una salida de campo a Medellín en la que, con ocasión delV Congreso Colombiano de Filosofia (28 de julio a $1^{\circ}$ de agosto de 2014) convocado por la Sociedad Colombiana de Filosofia y organizado por la Universidad de Antioquia y la Universidad Eafit, fueron entrevistados algunos exponentes del pensamiento kantiano en relación con nuestro tema de interés.

Los autores expresan su gratitud especial con el profesor Leonardo Tovar González, por su dirección, asesoría y acompañamiento constante en la investigación; y a las profesoras de la Facultad, Gloria Isabel Reyes y Ángela Niño Castro, por su permanente apoyo en las gestiones administrativas que demandó el desarrollo del proyecto. Asimismo, destacan el apoyo brindado por Camila Suárez, Julián Pacheco y demás funcionarios de la Unidad de Investigación y otras instancias de la Universidad. Manifiestan además un agradecimiento particular a la Sra. Luz Marina Páez y su equipo de colaboradores por haber facilitado para el desarrollo del proyecto, las instalaciones de la biblioteca "José de Jesús Farías Páez O.P." de la sede Aquinate de la Universidad.

Junto con los demás semilleristas que integraron el equipo investigativo (Robinson Rodríguez, Kevin Bautista, Juliana Granados, Elizabeth Herrera), dedicamos esta incipiente indagación, a las filósofas y filósofos colombianos que han anticipado esta preocupación de reflexionar con ojos kantianos sobre nuestra situación, y con ojos colombianos sobre el pensamiento de Kant.Van en especial nuestros reconocimientos a los maestros que de manera generosa atendieron la invitación a conversar sobre Kant y la paz en Colombia.

\section{LA PAZ EN PERSPECTIVA DEL DERECHO PÚBLICO}

El concepto de paz que desarrolla el pensador de Königsberg nos remite, en un primer momento, a la idea de una sociedad consolidada por el derecho, el cual guía las acciones de los individuos tanto en su condición de gobernantes como de súbditos, en favor de procurar la Federación 
de Estados, garante del proyecto de la paz. Así, de acuerdo con esta noción de derecho, la moral y la política entran en constante diálogo, pues no debe ser más que la razón la que guíe lo que se debe hacer en situaciones de hostilidad y peligro. En este contexto, se manifiestan diferentes posiciones acerca de la influencia de la moral sobre la política, ya que pueden encontrarse dirigentes que menosprecian la labor de la moral, permitiendo que sus intereses prevalezcan sobre el bien de los demás. A ellos se les reconocen como moralistas políticos. En cambio, los que tienen como recto orientador a los principios morales en su ejercicio político, se los llama políticos morales; estos tienen por cualidad propia, poder reflexionar y decidir correctamente lo que es más conveniente y mejor para el Estado que gobiernan. Así, la prevalencia de la moral sobre la política se aclara aún más cuando Kant establece en el Opúsculo de la paz que:

[...] las máximas políticas no deben partir del bienestar y de la felicidad que cada Estado espera de su aplicación, que no deben partir, por tanto, del fin que cada Estado se propone, que no deben partir de la voluntad como supremo (aunque empírico) principio de la sabiduría política sino que deben partir del concepto puro del deber jurídico, sean cualesquiera las consecuencias físicas que se deriven (el deber, cuyo principio está dado a priori por la razón pura). (Kant, 2010, p. 704)

Por esta razón, el derecho debe ser concebido en cualquier Estado como el fundamento del quehacer político, cuya realidad empírica no se vería de ninguna forma limitada. Antes bien, el derecho en sí mismo está abierto a ella en el ejercicio de valerse de la propia razón en comunidad con otros, de tal manera que cuando el individuo hace uso público de la razón respecto, no sólo a asuntos personales, sino a los que incumben a otros, pone sobre la mesa ante el público racionizante aquello que se puede deliberar entre todos y que no solo queda en las manos de quien gobierna; lo cual puede resultar riesgoso al juzgar imparcialmente y de manera injusta, sin tener en cuenta a las otras partes.

Entendemos de este modo que las disposiciones de carácter político, que intervienen directamente en el proyecto de la paz perpetua que plantea Kant, tienen un trasfondo moral innegable que determina todo 
su proceder. Con esto, el derecho mas allá de ser un compendio de estatutos para vivir en comunidad es, en consonancia con la moral, una norma universal, que a pesar de ser anterior a la experiencia del hombre en la sociedad, lo determina en su actuar dentro de esta. De ahí que la ley jurídica se ciña a los dictámenes de la razón, pero sea instaurada no por el mismo sujeto sino por una imposición de carácter externo.

Asimismo, centrándonos más en lo que concierne a la paz, el pensador alemán estipula que es un deber procurar un Estado de derecho público que posibilite emprender la tarea infinita de esta, cuyo resultado inmediato no debe ser el más eficaz pues los tratados de paz, al ser meras conciliaciones, dejan abierta la posibilidad de más guerra y conflicto entre los Estados y al interior de ellos. Este debe ser internamente estable y tener un buen funcionamiento para unirse a otros y procurar esa inevitable esperanza de la paz, que ciertamente, según Kant, genera un progreso inalcanzable de la humanidad hacia lo mejor.

En este orden de ideas, en el texto "Idea de una historia universal en sentido cosmopolita", Kant plantea una serie de principios donde reflexiona acerca de la idea de una historia de la humanidad en general, que concibe como un curso progresivo que lleva al hombre, sin percatarse de aquello como individuo sino sólo como miembro racional de la especie humana, a la realización de la intención que la Naturaleza le constriñe. Por muy azarosas que puedan parecer las acciones, si se les mira desde un panorama global, dicha intención se manifiesta a la especie humana como un motor regulador que la orienta a la conformación de una "sociedad civil que administre el derecho en general" (Kant, 2010, p. 19). Es decir, que la Naturaleza desea para el hombre, gracias a la disposición natural que tiene como ser racional, la conformación de un Estado bajo las normas de una constitución civil (Kant, 2010).

Dice Kant, en el tercer punto del suplemento primero del opúsculo de la paz, que "la Naturaleza garantiza la paz perpetua" (2010, p. 704), dado que la razón utiliza el mecanismo natural de la insociabilidad como medio para alcanzar su fin propio, el precepto legal (Ak VIII $367 \backslash$ p. 692). La insociabilidad no es más que la condición inmanente del individuo como ser ambicioso, competitivo, egoísta, que lo dirige constantemente hacia un perpetuo conflicto con los demás. Sin embargo, sólo a partir de esta 
naturaleza intrínseca y de las consecuencias nefastas que puede acarrear, la razón obliga a la voluntad de los hombres a consensuar y formar una sociedad civil donde las acciones fundamentadas en la libertad sin ley sean limitadas por el derecho. Pero por otra parte, aclara Kant que la naturaleza, si bien no garantiza en un sentido teórico el progreso de la humanidad hacia la paz, "sí resulta suficiente y convierte en un deber el trabajar con miras a este fin (en modo alguno meramente quimérico)" (Kant, 2010, p. 693).

La paz entonces sólo es posible como idea regulativa que proyecta a la humanidad hacia el fin que la razón le impone a su voluntad, la sociedad civil, fundamentada en el derecho, como solución que permite al hombre tener una "vida digna" (Kant, 2010, p. 20). No obstante, dicha imposición natural (que no limita la libertad práctica) está dirigida a la voluntad colectiva de los hombres, según Kant, pero no a la voluntad particular de cada individuo porque, ciertamente, el hombre en su particularidad no está impulsado por ningún precepto moral que guíe sus acciones, sino que remata en todo lo contrario, en su condición insociable para manifestar su desembarazada libertad. Por esta razón, sólo en la medida en que todos los hombres quieran establecer una sociedad civil bajo una voluntad general, consensuada, que trascienda la individualidad, podrán convivir de tal manera que cada quién tenga una vida digna.

Cabe anotar que precisamente por esta condición colectiva de la paz, el propósito de la razón sólo puede desenvolverse en la especie humana y no en los individuos en particular (Kant, 2010). Esto garantiza que el hombre, en su individualidad, al no recibir de ésta alguna utilidad que afecte su inmediatez, no necesariamente deba menospreciar la idea de la paz cuando tiene por ella una utopía fantasiosa propia de los cuentos de hadas. Es por esto que el hombre, si quiere alcanzar un estado de paz, debe tener claro que esta idea posiblemente nunca se materializará en su entorno a pesar de su trabajo incesante. Lo que no significa, sin embargo, que por ello perderá la obligación de trabajar para conseguir su realización.

Dice Kant que el hombre, en su condición de ser mortal, no cuenta con el tiempo suficiente de vida para desarrollar todas sus facultades, entre ellas la facultad de razonar -cuyo uso práctico apunta a la consolidación de un estado de dignidad- lo que finalmente lo llevará a necesitar de una "serie incontable de generaciones que se transmitan una a otra sus conocimientos" 
(Kant, 1985, p. 43) Es menester para el hombre, según lo anterior, no perder confianza en las acciones encaminadas a la construcción de una sociedad civil, pues aunque no se alcance a experimentar en un nivel pragmático todos los beneficios que pueda acarrear, que según Kant es lo más probable, sí y solo sí es obligación de nosotros continuar con ese proyecto, de tal manera que una próxima generación pueda recoger los frutos de la anterior y llevar a cabo ese propósito que la naturaleza tiene para el hombre.

El concepto de paz será entonces: por un lado, el estado de dignidad perfecto que posiblemente nunca se manifestará entre nosotros, pero que por otro, referirá a una idea que regule nuestras acciones dentro de una sociedad civil. Acciones fundamentadas en el derecho jurídico que ponga límites a nuestra libertad sin ley, de manera que cumpliendo lo que impone la razón a la voluntad, actuemos consecuentemente para que, al menos, podamos disfrutar de una vida que participe de dignidad, donde nuestra libertad esté delimitada por la libertad de aquellos con los que convivimos. Esta característica suprime de la paz el carácter utópico fantasioso que pueda representar, ya que la paz, en este caso, no se reduce a una ideología justificadora por la que debamos anular nuestra individualidad, nuestra propia libertad, para trabajar por la realización de una felicidad ajena, sino como idea regulativa que se fundamenta en el derecho jurídico. Esta es la única defensa legítima que nos puede garantizar una vida digna tanto para los hombres de hoy como para las próximas generaciones.

\section{"LA PAZ PERPETUA" DESDE FILÓSOFOS COLOMBIANOS}

En este diálogo político sobre la paz desde una perspectiva Kantiana, encontramos dos lecturas políticas colombianas: el caso de Rubén Jaramillo, que hace énfasis en lo histórico y el caso de Manuel Guillermo Rodríguez, que se concentra en lo revolucionario.

En el texto "Antecedentes de la paz perpetua" (2004) (con motivo del bicentenario) el filósofo colombiano Rubén Jaramillo Vélez realiza un seguimiento a varias reflexiones que llevaron a cabo algunos pensadores como Rousseau y Hobbes, los que en su punto conformaron la antesala de la publicación en 1795 del opúsculo de Kant "Sobre la paz perpetua”. Dicha publicación, escrita hace más de doscientos años, fue el fruto que nació del 
autor ante la preocupación por establecer unas condiciones mínimas para que los Estados permitieran al hombre convivir, si no en paz sí con dignidad.

El filósofo colombiano, en su texto, retoma algunas concepciones kantianas que se relacionan con el tema de la paz, tales como la tendencia moral del género humano que permite un constante progreso hacia lo mejor (Jaramillo, 2004), el mecanismo natural de la insociable sociabilidad, el plan oculto de la naturaleza que guía a los hombres para que consoliden una sociedad civil fundamentada en el precepto legal. En fin, conceptos que pueden ser una referencia para pensar el proyecto de la paz que tanto anhelamos en estos tiempos de conflicto.

Sin embargo, nos dice el profesor Jaramillo que luego de cumplirse el bicentenario de su publicación, "la paz perpetua" todavía permanece como proyecto en razón de los acontecimientos que hasta la fecha el hombre ha tenido que padecer, como el fascismo hitleriano y, efectivamente, el conflicto armado en Colombia. De esta manera, resulta de suma importancia para el semillero considerar la reflexión principal del texto acerca del proyecto de la paz kantiana expresado por nuestro profesor, en el cual menciona que el fracaso del proyecto de la paz durante doscientos años es consecuencia de querer eliminar del panorama toda amenaza de guerra, considerada por Kant como un grave crimen contra la humanidad (Jaramillo, 2004). Rubén Jaramillo, por otra parte, cree firmemente que sólo a través de una "política realista a favor de la paz, que no excluya ni reprima ni disminuya la verdadera naturaleza de los conflictos, de toda índole, que afectan tanto a los individuos como a los grupos, clases y naciones de nuestra sociedad planetaria, podrá garantizar el futuro que hace doscientos años avizoraba Kant" (2004, p. 41).

Es así como empezamos a escuchar la voz de nuestro primer filósofo, quien reflexiona y propone una nueva condición para que el proyecto de la paz kantiana tenga viabilidad: la materialización de una "política realista". Dicha política, debe estar caracterizada por el diálogo entre las partes como un camino a seguir para alcanzar la paz. Como bien sabemos, el rumbo que el actual gobierno colombiano ha decidido recorrer, a diferencia de gobiernos anteriores, ya no versa sobre la supresión y eliminación del otro, sino sobre la búsqueda de consenso que pueda dirimir el conflicto. Más adelante en este artículo, veremos qué tanto se corresponden 
las circunstancias del actual proyecto de la paz llevado en Colombia con esta propuesta de Rubén Jaramillo.

Crítico del dogmatismo religioso y político, el profesor Rubén cree firmemente en el progreso como un paso del Estado capitalista a un Estado socialista en el cual confluyen las ideas que proponen Lutero, Kant, Marx, Freud, entre otros, donde convergen en aquella máxima de la Ilustración que ve en el hombre un sujeto capaz de asumir la responsabilidad de pensar por sí mismo. Su objetivo entonces, consiste en realizar un análisis histórico desde el cual se pueda comprender por qué en Colombia la Ilustración, y entre ella la idea de paz, continúa siendo un proyecto postergado acomodado en concepciones heredadas de la época colonial, y a partir de dicho análisis propone como salida la "política realista".

Asimismo, el pensamiento político kantiano ha sido uno de los ejes de interés y estudio de su discípulo, el profesor Manuel Guillermo Rodríguez. Según desde su común opción por Marx, Rubén Jaramillo Vélez y Manuel Guillermo Rodríguez se relacionan en una lectura política de la propuesta Kantiana aplicada a nuestra situación. Por su lado, el primero, como ya dijimos, hace énfasis en un análisis histórico del proyecto postergado de la paz. Por el otro, el segundo autor, en su artículo "La paz perpetua, un esbozo filosófico" (2004) apunta a una transformación política de la sociedad desde las estructuras socioeconómicas, en donde la revolución teórica propuesta por Kant sería un antecedente de la revolución marxista materialista.

No obstante, teniendo en cuenta que la conciencia de la modernidad permitió que las limitaciones del modelo feudal desaparecieran para desarrollar la autonomía y la libertad tanto en el individuo como en la sociedad, existen teorías que han tratado este tema en vista ya sea de un cambio político o con el fin de mantener un discurso a partir de tales categorías. Es así como el profesor Rodríguez desarrolla dos ideas: la primera se refiere al paso que hace el hombre del estado de naturaleza a la historia; la segunda, hace alusión a la relación que existe entre moral y política. Así pues, desde la lectura que hizo Kant de los postulados políticos de Rousseau, acerca de la libertad y su articulación con las leyes establecidas, el filósofo de Königsberg empieza a considerar otra idea del estado de naturaleza, puesto que de acuerdo con la lectura del profesor Rodríguez (2004), para Kant el estado de naturaleza no es mejor que el Estado civil, 
porque el hombre para construir la paz necesita salir de aquel estado y vincularse por medio del derecho a la sociedad. Además, el paso del estado de naturaleza a la sociedad significa también que el hombre no se limite a su ordenamiento mecánico, sino que logre superarlo.

De ahí que el derecho posibilite el ejercicio de la libertad mediante las condiciones que desarrollan de forma pacífica las potencialidades humanas. Por tal razón, el derecho es el primer escalón para la construcción de la historia, ya que permite el paso del estado natural a la sociedad. De esta manera, la disposición de la naturaleza misma es que el hombre se vincule por medio de un contrato, que es la primera forma de libertad, a una norma común que le permita vivir con la paz necesaria que surge de la convivencia con los otros y le posibilite educarse, lo cual constituye una garantía para la posteridad. Tal norma común debe estar incorporada dentro de una Constitución Republicana que garantice y respalde la libertad, la dependencia y la igualdad.

A propósito de la paz y de su participación en la transformación de la sociedad, dicho postulado kantiano permite entrever, en primera instancia, una preocupación por la fundamentación de las revoluciones, su impacto social y las medidas que las autoridades deciden emprender para autorizarlas o desautorizarlas, a favor o no de un proyecto de paz. En consecuencia, Kant acepta que en ese paso dialéctico del mundo de la naturaleza al de la sociedad civil, "si un movimiento revolucionario provocado por una mala constitución, consigue instaurar ilegalmente otra más conforme al derecho, ya no podrá ser permitido a nadie retrotraer al pueblo a la constitución anterior" (citado por Rodríguez, 2004, p. 238). En segunda instancia, el reconocimiento, la reformulación y el análisis de categorías como la libertad, la autonomía, la paz, la guerra y las revoluciones, que encaminan el problema de la paz a un horizonte práctico, sin dejar de lado los principios teóricos de orden moral que fundamentan su quehacer político.

Ahora bien, frente a las perspectivas de estos dos filósofos, podríamos traer a colación la reflexión del profesor Saldarriaga presentada en la entrevista que realizamos. Allí, considera que los aportes kantianos al concepto de la paz efectivamente sí son vigentes en el ámbito académico, ya que tienen que ver con la observancia del derecho por parte de los ciudadanos, que en este orden de ideas, no se vincula con la moralidad, 
sino con el concepto de legalidad que debe existir cuando determinado país está en cierta condición de tranquilidad y aspira junto con otros Estados a proyectos más relevantes para la humanidad. Por tal motivo, concibe que la noción de dignidad y respeto que expresa Kant en su filosofia es pertinente en el caso colombiano, ya que se encuentra latente en la Constitución de 1991. No obstante, considera que tales aportes son insuficientes para abordar un conflicto como el de Colombia, puesto que para ello se necesita más que unas categorías de un solo filósofo y más que las reflexiones de una disciplina, que en este caso sería la filosofía. Así, la posición de nuestro entrevistado en referencia a la problemática del conflicto y la paz en Colombia, es que no sólo se necesita de la participación del ciudadano como académico y como ciudadano. Además, afirma que es "una situación en la cual convergen problemas teóricos, problemas estructurales, problemas históricos que... no se pueden solucionar desde una sola perspectiva; desde un solo enfoque" (Saldarriaga, 2014).

De manera que, más allá de las formulaciones académicas, hemos dado cuenta de la vigencia que tienen los conceptos kantianos en lo que se refiere al proyecto de la paz y la apuesta favorable de nuestros filósofos para alcanzar su realización. Esto se hace más evidente en las reflexiones del profesor Carlos Patarroyo concedidas en una entrevista en elV Congreso Colombiano de Filosofia. Para el semillero ha sido importante rescatar, por una parte, la preocupación del filósofo Carlos Patarroyo acerca de los afanes que se presentan en Colombia para establecer un acuerdo de paz en un mínimo de tiempo posible. Esta preocupación del filósofo es perfectamente comprensible, debido a que las presiones surgen con el propósito de lograr que el consenso entre las dos partes dialogantes en la Habana pueda materializar en la vida cotidiana de los colombianos los cambios más esperados: cese al fuego, liberación de secuestrados, justicia, perdón, reposición, etc. Sin embargo, este anhelo vehemente de acelerar los procesos, si bien es comprensible, manifiesta nuestro profesor, también puede ser causante del fracaso de los diálogos en la Habana en la medida que fuerza a encontrar soluciones rápidas a problemas de fondo (y no es para menos, ya que son problemas que han perdurado durante más de cincuenta años) cuyo propósito, en últimas, obligaría a cumplir fechas preestablecidas y ceder a presiones externas para dar cuenta de la efectividad 
política del gobierno de turno. Lo que en la perspectiva del profesor Jaramillo vendría a ser una especie de falacia de aquella política realista que propone, puesto que el gobierno, decorando el verdadero acontecer del conflicto, se propone lograr consenso bajo la etiqueta de diálogo que a la larga no desembocará sino en la perpetuación del problema.

Inquietado por esta problemática, el profesor Patarroyo señala, muy kantianamente, que los colombianos debemos tener la paciencia que requieren estos procesos aun si los cambios acordados, más sólidos entonces, no afectan directamente a los habitantes de la Colombia de hoy, sino que sean los frutos que una próxima generación pueda recoger.

El doctor Patarroyo, por otra parte, nos expresa que en el trasfondo del proyecto de la paz está la idea de humanidad, idea que nos permite tomar a los hombres como fines en sí mismos, como telos, y no como argumentos ad hoc. Esta idea de humanidad, dice el filósofo en la entrevista, es una idea regulativa -tal como la idea de paz- y que por lo tanto, se convierte en un proyecto inalcanzable. No obstante, nos dice que esto no resta importancia al trabajo que debemos realizar para alcanzarla, ya que el empeño puesto en todas las acciones que concretemos en nombre de esta idea regulativa no resultarán vanas y absurdas, pues a pesar de no recibir beneficios pragmáticos en nuestra vida cotidiana, sí estaremos contribuyendo, como seres racionales pertenecientes a la especie humana, a un progreso que apunta hacia la consolidación de un estado que permita vivir con dignidad.

En el trasfondo del proyecto de paz, nos dice el filósofo colombiano, está presente una idea regulativa que dirige nuestras acciones en provecho de la sociedad civil. Es decir, en provecho tanto para las personas con las que convivimos como para nosotros mismos, pues es una idea fundamentada en el derecho jurídico que limita nuestra libertad frente a la libertad de los demás. La idea de humanidad hace que nuestras acciones que tienen correspondencia con el derecho jurídico, no sean, tal como dice, vanas y absurdas, pues independiente de los resultados inmediatos que pueda acarrear una idea de paz, cumplir la obligación de tratarnos como hombres, es decir, como fines en sí mismos y no como medios útiles para conseguir algo, es una responsabilidad que dignifica la vida perfectamente justificada por el derecho jurídico. 


\section{LA PAZ: LECTURAS DE KANT Y SU HABER EN EL CONFLICTO COLOMBIANO}

Acorde con el pensamiento del profesor Rubén, se considera que en parte es la "política realista" la faltante para que el anémico proyecto de la paz kantiana evite postergarse indefinidamente, lo que daría una voz de aliento a quienes esperan que los diálogos de la Habana brinden las condiciones necesarias para que la paz pueda ser un proyecto realizable en Colombia. Por lo demás, podemos pensar que en el gobierno actual se ha implementado dicha política en la medida que ha reconocido la existencia del conflicto armado en Colombia. Reconocer el conflicto armado es, según la cita a Arturo Mujica que se hace en el artículo de la sección de Nación de la revista Semana: ¿¿Qué significa el reconocimiento del conflicto armado por parte del Gobierno?” (2011) “el primer reconocimiento político concedido por el gobierno en cuanto conflicto interno se refiere, pues en gobiernos anteriores lo único que se reconocía era una 'amenaza terrorista"”.

Si lo ponemos en términos de conflicto armado y basados en la definición que nos da el diccionario para desarmar la palabra, el Estado reconoce entonces la existencia de una oposición política que utiliza la fuerza bélica para imponer su forma de gobierno, condición que resulta clave para la posibilidad de establecer un diálogo que permita zanjar el conflicto político por vías diferentes a la guerra armada; caso contrario ocurre cuando el Estado determina el conflicto como efecto de grupos terroristas, tal como lo menciona un expresidente de Colombia: "los grupos narco-terroristas colombianos no son partes en conflicto. Son criminales que perfuman sus acciones con discursos políticos" (Revista Semana, 2011). En tal caso, la posibilidad de diálogo quedaría excluida para dar paso a una política de exterminio y represión de los grupos insurgentes, movidos por ideologías distintas.

Vemos entonces que la condición necesaria presentada por el profesor Rubén Jaramillo para que el proyecto kantiano de la paz se lleve a cabo, está en sintonía con las circunstancias actuales del gobierno -en torno a este punto- y con su disposición a dialogar. A esto se le añade, por otra parte, la fuerte oposición que se gesta desde las corrientes políticas adversas al proceso de paz, además de la clara resistencia que se ejerce desde el Ministerio Público, porque en el debate de este proyecto no 
sólo deben participar aquellos afines al proceso (con carácter privado) sino que debe manifestarse, en un ámbito más conforme con el derecho público, donde sean posibles la confrontación permanente de todas las instituciones y de todos los ciudadanos en general; y no como las acciones efectuadas por algunas ideologías oscuras que abanderan, entre otras, políticas de rechazo, como el comentario citado del expresidente; de privacidad, como el delito que se le atribuye a un magistrado de la corte constitucional (El tiempo, 2016); y de exclusión o segmentación, como la propuesta de dividir el departamento del Cauca (El espectador, 2015).Así pues, desde esta perspectiva "política realista", se muestra que están prestas las condiciones para que en el proyecto de la paz no siga perpetuándose el fracaso. Sin embargo, en el tiempo que lleva el proceso han ocurrido contratiempos que revelan ciertas incongruencias con el derecho, tal y como sucedió con el secuestro de un general de las fuerzas armadas legítimas (El tiempo, 2014). En este acontecimiento, tan importante para la historia de Colombia y en especial para el Ejército Nacional, salieron a flote circunstancias inesperadas, que desde un punto de vista filosófico kantiano, pueden representar cuestiones adversas al proceso de paz.

Por un lado, vimos que la primera reacción del presidente fue exigir a los altos mandos del Gobierno la explicación de por qué el general violó los protocolos de seguridad (El Tiempo, 2014). Lo que significa que el presidente, tal como lo dice un jefe guerrillero en una carta escrita al general luego de su liberación: "antes que expresar algún tipo de preocupación por la vida o la libertad de un general, había que salir a exigir explicaciones sobre sus motivos para estar exponiéndose de tal modo" (Semana, 2014). La reacción del presidente es precisamente un comportamiento característico de lo que Kant llamaría un "moralista político", ya que dio prelación a los intereses políticos del Gobierno frente a la acción política fundamentada en el derecho, es decir, que la vida del general y de los otros secuestrados fue subyugada por la "humillación al Ejército" que generó el inconveniente con el grupo guerrillero.

Otra forma de evidenciar las incongruencias con el derecho jurídico, fue cuando el presidente, a pesar de haber suspendido las negociaciones de paz exigiendo como condición de reapertura la liberación pública de los secuestrados, de una forma secreta planeó alternativas de liberación. 
Dice en la carta, publicada por El Espectador el martes 02 de diciembre de 2014, del jefe guerrillero Timochenko "es claro que no se trata igual a un general que a unos soldados" (2014). Se evidencia entonces que las acciones políticas del presidente tampoco estaban fundamentadas en el derecho, ya que sus decisiones fueron movidas por intereses alternos a la exigencia por el respeto a la vida y a la libertad de los seres humanos.

Si lo abordamos desde este punto de vista, es preciso traer a colación el comentario que realizó el procurador general de la nación en un enfrentamiento público con el presidente de la República: “el proceso [diálogos en la Habana] no está por encima de la Constitución, ni el presidente por encima de la ley, ni siquiera con la excusa de la paz" (El país, 2015). Con este comentario, muy pertinente para el semillero, es claro que, aunque no son delitos sancionados por la justicia penal, y con referencia a los ejemplos anteriores, el presidente no actuó conforme a la ley. Lo que llama la atención en este punto es que el propósito de lograr la paz se puede convertir en una idea que justifique todas las acciones incluso si aquellas pasan por encima del derecho, de manera que el moralista político -en este caso el presidente- "subordina los principios al fin, es decir, engancha los caballos detrás del coche" (Kant, 2010 p. 702).

La condición necesaria que posibilite una salida favorable al proyecto de la paz y que, sin embargo, no permita un abuso del derecho, considera el semillero, fue planteada por el ex secretario general de la ONU, Kofi Annan, durante su visita a Colombia: "la justicia no puede ser un impedimento para la paz, es un socio absolutamente esencial para ella" (El Espectador, 2015) en el cual se manifiesta "la importancia de encontrar un punto de equilibrio que no sacrifique la justicia, pero que tampoco deje en vilo el proceso de paz" (2015). Solo de esta manera, ciertamente, la paz no se convertirá en la idea que justifique acciones impunes encaminadas hacia el fin propuesto por una élite y pueda llevarse a cabo, efectivamente, el desarrollo de aquella "política realista" habilitada para dirigir correctamente las acciones en torno a esa idea de paz que todos los colombianos anhelamos.

A modo de conclusión, vemos que la actual coyuntura colombiana en relación al proceso de paz, si bien está lejos de alcanzar ese estado perfecto en cual todos los ciudadanos sean tratados con dignidad, y más lejos todavía de lograr un Estado firme del que sea posible esperar "la formación 
moral de un pueblo (...) incluso para un pueblo de demonios" (Kant, Ak VII 366\ p. 691), nos autoriza a pensar que las actuales condiciones para la resolución del conflicto posibilitan en mayor medida lograr resultados favorables para todos los ciudadanos. Esto no sólo gracias a la buena disposición del gobierno y de las FARC para reconocerse como oponentes políticos y conciliar sus diferencias, sino gracias también al carácter público que permite la crítica constante desde otras perspectivas, incluyendo a los directamente afectados, es decir, a las víctimas, y a los oponentes ideológicos.

Finalmente, son muchas las cosas que podemos decir sobre el proceso de la Habana si tenemos como referencia tanto al filósofo alemán como a los filósofos colombianos. No obstante, lo verdaderamente importante que podemos resaltar aquí es el optimismo de nuestros filósofos colombianos por este acontecimiento y la apuesta positiva por un cambio favorable a toda la sociedad.

Nosotros, como semillero de investigación interesado en la filosofia de Kant, nos ubicamos del lado de aquellos que piensan que lo mejor para el país es buscar la paz sin importar cuáles son las condiciones que están de por medio para experimentarla. Pese a que muchos, incluso el mismo Kant, ven al proyecto de la paz como un ideal imposible de realizarse, debemos estimarla como ese ideal regulativo que orienta nuestras acciones y las dirige siempre hacia la constante afirmación de los hombres como seres humanos.

\section{REFERENCIAS}

El Espectador. (Fecha del artículo día, mes y año 2014). ‘Timochenko' envía carta abierta al general Alzate. Recuperado de: enlace.

El Espectador. (16 de marzo de 2015). Paloma Valencia propone dividir el departamento del Cauca. Recuperado de https://www.elespectador.com/noticias/nacional/ paloma-valencia-propone-dividir-el-departamento-del-cau-articulo-549804

El Tiempo. (17 de noviembre de 2014). El general Rubén Darío Alzate Mora fue secuestrado en Chocó. Recuperado de http://www.eltiempo.com/archivo/ documento/CMS-14840497

El tiempo. (25 de agosto de 2016). Jorge Pretelt, primer magistrado que va a juicio ante la Corte Suprema. Recuperado de http://www.eltiempo.com/politica/congreso/ jorge-pretelt-primer-magistrado-que-va-a-juicio-ante-la-corte-suprema-35968 
El País. (28 de marzo de 2015). Nuevo choque del Presidente y el Procurador por el proceso de paz. Recuperado de http://www.elpais.com.co/colombia/nuevochoque-del-presidente-y-el-procurador-por-el-proceso-de-paz.html

Jaramillo, R. (2004) Antecedentes de la Paz Perpetua (con motivo del bicentenario). En: "Kant y la paz perpetua: homenaje en el bicentenario de la muerte de Kant (1804-2004)” Colombia: Ediciones Jurídicas Gustavo Ibáñez.

La Prensa. (2015). Juan Manuel Santos y Alejandro Ordóñez se enfrentan por diálogo de paz con las FARC.

Kant. (2010). Hacia la paz perpetua: un esbozo filosófico. (J. M.Veiga, Trad.) Madrid: Gredos.

Kant, I. (2010). Ideas de una historia universal en clave cosmopolita. En KANT (R. Rodríguez Aramayo, Trad., Cuarta ed.,Vol. II, págs. 13-27). Madrid: Gredos.

Rodríguez, M. (2004). La paz perpetua, un esbozo filosófico. En: "Kant y la paz perpetua: homenaje en el bicentenario de la muerte de Kant (1804-2004)" Colombia: Ediciones Jurídicas Gustavo Ibáñez.

Saldarriaga, A. (1 de agosto de 2014). Comunicación personal. Entrevista realizada por María Parra Toquica.

Revista Semana. (5 de abril de 2011). ¿Qué significa el reconocimiento del conflicto armado por parte del Gobierno? Recuperado de http://www.semana. $\mathrm{com} /$ nacion/articulo/que-significa-reconocimiento-del-conflicto-armado-parte-del-gobierno/239313-3 
